\title{
Ethical, legal and social implications of susceptibility genetic testing for late-onset neurodegenerative diseases
}

Amaranta Manrique de Lara1 ${ }^{1}$, Elisa Núñez-Acosta² ${ }^{2}$ Garbiñe Saruwatari-Zavala³ ${ }^{2}$ Liliana Soto-Gómez ${ }^{4}$ and Miguel E. Rentería ${ }^{5}$

1Licenciatura en Ciencias Genómicas, Instituto de Biotecnología y Centro de Ciencias Genómicas, Universidad Nacional Autónoma de México, Cuernavaca, Morelos, México. E-mail: amanriqu@lcg.unam.mx

ºficina de Información Científica y Tecnológica para el Congreso de la Unión (INCyTU), Foro Consultivo Científico y Tecnológico, A.C., Coyoacán, Coyoacán, Ciudad de México, México. E-mail: e_nunez@foroconsultivo.org.mx

3Instituto Nacional de Medicina Genómica, Tlalpan, Ciudad de México, México. E-mail: gsaruwatari@inmegen.gob.mx

4Instituto de Investigaciones Jurídicas, Universidad Nacional Autónoma de México, Coyoacán, Ciudad de México, México. E-mail: lily_sg84@hotmail.com

5Department of Genetics \& Computational Biology, QIMR Berghofer Medical Research Institute, Brisbane, QLD, Australia. E-mail: miguel.renteria@qimrberghofer.edu.au

Correspondence: Miguel E. Rentería, QIMR Berghofer Medical Research Institute, Locked Bag 2000, Royal Brisbane Hospital, Brisbane, Queensland 4029, Australia.

E-mail: miguel.renteria@qimrberghofer.edu.au.

\begin{abstract}
(200 words):
Over the last decade, advances in our understanding about the genetic architecture of complex traits and common diseases, have increased our ability to perform susceptibility genetic testing for diseases in asymptomatic individuals. These technological developments raise complex ethical, legal and social considerations. Here we discuss a series of ethical issues associated with susceptibility genetic testing for Alzheimer's and Parkinson's disease. These include, amongst others, informed consent, disclosure of results and unexpected findings, mandatory screening, privacy and confidentiality, and stigma and genetic discrimination. As knowledge of the genetic basis of these diseases continues growing, and as genetic testing becomes more widespread, we anticipate that it will become increasingly important for scientists and clinicians to engage in the conversation about the ethical, social and policy implications of these technologies.
\end{abstract}


Keywords (3-5): Alzheimer's disease, Parkinson's disease, Genetic testing, Bioethics.

\section{Introduction}

Genetics research has changed dramatically since the sequencing of the human genome, increasing its focus on common diseases rather than single gene disorders. Complex disorders, such as diabetes, asthma, coronary heart disease or schizophrenia, result from the contributions of multiple genes and environmental factors. Over the last decade, genome-wide association studies (GWAS) and large-scale international genetics consortia have facilitated the discovery of thousands of associations between common genetic variants and complex traits and diseases. This unprecedented progress in human genetics has enabled the possibility of susceptibility genetic testing, using methods such as polygenic risk scores (PRSs), which can estimate the relative genetic susceptibility of an individual to develop a disease based on their genetic profile.

Alzheimer's disease (AD) and Parkinson's disease (PD) are two highly debilitating late-onset neurodegenerative diseases. There is currently no cure or effective treatment for either, and their prevalence is expected to increase with population ageing. Due to their considerable personal and economic costs, they pose an important challenge to societies around the world. Recent advances in biomedical science, and especially in neurogenetics, offer great hope for improving the prevention, diagnosis and treatment of both $\mathrm{AD}$ and PD. In particular, the power of genetic testing to identify individuals with increased susceptibility, and the ability to convey information about their relatives, create a set of complex ethical, legal and social issues. Here, we aim to contribute to the debate, discussing the issues related to the use of genetic testing to assess susceptibility to late-onset neurodegenerative diseases in asymptomatic individuals and its impact on different social spheres.

\section{Late-onset neurodegenerative disease in the genomics era}

This first section will briefly detail the underlying mechanisms, pathology and genetic components of each of the two most common neurodegenerative disorders: Alzheimer's and Parkinson's disease. In addition, we will detail their incidence and prevalence, and the resulting global economic impact. Finally, we will outline how genomic technologies have and will impact the clinical handling of AD and PD.

\subsection{Alzheimer's disease}


Alzheimer's disease is a chronic and progressive disorder which leads to cognitive impairment and neuropsychiatric abnormalities. It is characterized by the accumulation of insoluble forms of the amyloid beta peptide $(\mathrm{A} \beta)$ and aggregation of tau proteins in wide areas of the cerebral cortex and the hippocampus.

$\mathrm{AD}$ is considered the most common neurodegenerative disorder, with an estimated prevalence of 10-30\% in the global population over 65 years of age (Masters et al., 2015). While evidence suggests that sporadic $\mathrm{AD}$ affects all of the world's geographical populations in similar rates, high-income countries currently account for about half of the global prevalence (Alzheimer's Disease International [ADI], 2010). Importantly, dementia seems to be more prevalent in women than men (Masters et al., 2015). Alzheimer's can dramatically shorten life expectancy: median survival with AD is approximately 7.1 years after onset (Duthey, 2013). It is it is difficult to determine the independent contribution of Alzheimer's to mortality, since people suffering from $\mathrm{AD}$ often develop comorbid health conditions, related or not to the dementia process itself.

In 2010, the cost of dementia per year was estimated at more than 604 billion US dollars, representing approximately $1 \%$ of the global Gross Domestic Product (Duthey, 2013; ADI, 2010). High-income countries account for $89 \%$ of costs, while developing countries contribute under $1 \%$ to total worldwide costs (ADI, 2010). This discrepancy is explained by the disproportionate reliance on informal (i.e. unpaid care provided by family) versus formal care (i.e. paid care provided by professionals) in different regions of the world. For example, 80-99\% of people with dementia in urban areas in Mexico live at home, with this number increasing to $95-100 \%$ of patients in rural areas. In contrast, just $50-69 \%$ of people with AD live at home in all areas of Australia (ADI, 2010).

Based on predicted increases in the global prevalence of dementia, partly due to the ageing population, costs of dementia in 2030 are expected to be $85 \%$ higher (ADI, 2010). Just in Europe, estimates of the future cost of $\mathrm{AD}$ predict a rise of $43 \%$ (Duthey, 2013). However, rising costs in developing economic regions are expected to be much sharper, because increased life-expectancy related to development will result in higher levels of AD. For example, the prevalence of dementia in Latin America is expected to rise by $435 \%$ by 2050 in relation to 2010 (ADI, 2010).

The vast majority of $\mathrm{AD}$ patients have the sporadic form of the disease, characterized by late onset; a small proportion of patients present early-onset inherited forms. A large number of genetic factors have been identified which determine the development of sporadic and inherited Alzheimer's. Familial AD follows an autosomal dominant pattern of inheritance, involving three main genes implicated in the genesis of $A \beta$ : presenilin 1 (PSEN1), presenilin 2 (PSEN2) and amyloid precursor protein (APP) (Verlinsky et al., 2002). Late-onset $\mathrm{AD}$ is much more genetically complex. The most common risk allele is found within the gene 
coding for apolipoprotein E (APOE), which is a major determinant of age of onset (Escott-Price, Shoai, Pither, Williams, \& Hardy, 2017). Depending on its isoform (APOE2, APOE3 or APO4), the risk of developing Alzheimer's increases or decreases. For example, one allele with the APOE4 polymorphism imparts a threefold increase in risk, while APOE2 is considered a protective factor (Masters et al., 2015). Other genetic determinants are involved in $A \beta$ clearance pathways. Overall, approximately 20 common loci have been identified as susceptibility factors for sporadic AD, and several more show some evidence of association (Escott-Price, 2017). Importantly, the genetic contributing factors we have described have been primarily discovered amongst European ancestry populations, as is the case for many diseases. For example, the rate of decline has not been consistently shown to be linked to the effect of APOE4 in non-white populations (Marden et al., 2016) and also differs across gender (Goldman et al., 2011).

Other risk factors have been identified in addition to the genetic component. For example, diabetes, depression, smoking and low levels of education have been correlated with increased AD risk (Masters et al., 2015). This highlights the relevance of environmental factors on the development and progress of complex disease.

\subsection{Parkinson's disease}

Parkinson's disease is a slowly progressing neurodegenerative disorder known for affecting movement. The most common motor symptoms include tremors, rigidity and bradykinesia (Farlow, Pankratz, \& Wojcieszek, 2004). Non-motor symptoms and comorbid conditions are also prevalent, such as cognitive decline, depression, dysphagia, and symptomatic postural hypotension (von Campenhausen et al., 2011). PD is characterized by the loss of dopaminergic neurons, partly due to the accumulation of the $\alpha$-synuclein protein (Poewe et al., 2017).

Parkinson's is the second most prevalent late-onset neurodegenerative disorder worldwide, with an overall prevalence of $1 \%$ of the over-60 population (de Lau \& Breteler, 2006). Importantly, the number of PD patients is expected to double between 2005 and 2030 (Poewe et al., 2017). In contrast to AD, Parkinson's disease is more prevalent in men than women (Poewe et al., 2017). Similarly, evidence suggests that race and ethnicity might have an important effect on disease incidence (Poewe et al., 2017). However, it is currently difficult to discern between purely biological causes and sociodemographic factors related to health (Roberts \& Uhlmann, 2013).

PD is considered one of the costliest diseases in some countries (von Campenhausen et al., 2011), as it represents a high economic burden on the patients, their close family, and society as a whole. In the United Kingdom, the expenditure on PD is estimated to be between 449 million and 3.3 billion pounds every year 
(Findley, 2007). One important characteristic of a neurodegenerative disease such as PD is that the cost escalates in proportion to the progression of the disease. Direct costs, such as inpatient care and medication, increase with the development of comorbid conditions and the need for more healthcare resources. Similarly, the loss of independence of the patients results in increased indirect costs, shown by decreased productivity.

Parkinson's disease exists in several forms, both hereditary and non-hereditary. Familial forms of PD are monogenic, inherited in autosomal dominant, autosomal recessive, or even X-linked patterns (Farlow et al., 2004). Sporadic or idiopathic forms of PD are more common and are caused by the cumulative action of multiple genes. Studies confirm that some of the genes associated with familial PD are also affected in the sporadic disease (Poewe et al., 2017). For example, mutations in the LRKK2 gene represent a frequent cause of all forms of Parkinson's, accounting for $1 \%$ of sporadic cases and $4 \%$ of familial cases (Kalia \& Lang, 2015). Risk-altering polymorphisms for sporadic disease can be found in PARK loci (initially discovered in familial PD), along with several other genes (Ibanez et al., 2017). Mutations in GBA, for example, are the most common genetic risk factors (Poewe et al., 2017). Overall, more than 26 independent single-nucleotide polymorphisms (SNPs) have been identified (Paul, Schulz, Bronstein, Lill, \& Ritz, 2018). Genetic factors contributing to disease are mainly associated with pathways related to $\alpha$-synuclein proteostasis and degradation, mitochondrial function and immune regulation (Poewe et al., 2017).

In addition to genetic factors, environmental and lifestyle influences over disease development and progression are also relevant. For example, exposure to pollutants, physical activity, vitamin D exposure and urate levels might contribute to overall risk of PD (Kalia \& Lang, 2015). Arguably, this might be due to environmental effects over the epigenetic profile of the nervous system (Poewe et al., 2017).

\subsection{Impact of new technologies on the clinical handling of AD and PD}

Accurate clinical diagnosis and treatment of neurodegenerative diseases such as AD and PD is a great challenge, given the complexity of said conditions. This is due to the difficulty of measuring and integrating all the factors which influence the expression of a disease across a patient's lifespan and differentiating these diseases from other disorders and common comorbidities. For example, over one third of clinically diagnosed patients with Alzheimer's disease in a large clinical study were later found to be misdiagnosed (Masters et al., 2015). Similarly, a systematic review of accuracy of clinical diagnosis of Parkinson's estimated a pooled diagnostic accuracy of $80.6 \%$ in 11 studies over the past 25 years (Rizzo et al., 2016). Importantly, accuracy has not significantly improved, particularly in the early stages of disease. 
New technologies, such as imaging and biomarkers, have emerged as essential tools to aid in the diagnosis of neurodegenerative disorders. Several biomarkers in the cerebrospinal fluid (CSF), for instance, are promising diagnostic tools for $\mathrm{AD}$, which could potentially enable diagnosis even before the development of clinical symptoms (Masters et al., 2015). Further, CSF-based diagnostic tests have also been suggested for PD (Poewe et al., 2017).

Genetic testing is another tool used to estimate susceptibility and aid diagnosis for several diseases, as well as an important tool for research. Genetic tests are sometimes used to assess multiple diseases at once, instead of being targeted for a specific condition. Depending on the disease of interest and the purpose of the test, a couple of main questions must be addressed to establish its usefulness: which gene (or genes) should be analyzed, and whether the test has sufficient sensitivity and specificity (Cozaru, Aşchie, Mitori, Poinăreanu, \& Gorduza, 2016). In the case of complex diseases such as AD and PD, genetic testing often yields ambiguous results. This is partly because the effect of the multiple genetic mutations can be altered by factors such as penetrance, expressivity, pleiotropy, epistasis or other known or unknown regulatory interactions (Roberts \& Uhlmann, 2013). In fact, genetic testing for complex diseases assesses susceptibility rather than being predictive, meaning that it is mostly probabilistic and highly contingent (Arribas-Ayllon, 2011).

Inheritance and susceptibility for $\mathrm{AD}$ and $\mathrm{PD}$ is difficult to predict, especially if only a single variant is assessed. Polygenic risk scores summarize genotype data to inform about the genetic architecture of a complex trait or a disease (Lewis \& Vassos, 2017). Each score is based on known risk and protective alleles in genome-wide data, and it is calculated from GWAS summary statistics for the trait of interest (Lewis \& Vassos, 2017). Discovery GWAS have estimated effects and effect sizes for hundreds of thousands of common variants, in addition to genome-wide significant loci. These additional polymorphisms also account for a fraction of the heritability of the disease and act in an additive manner (Escott-Price et al., 2017; Poewe et al., 2017).

The PRS is a powerful tool, precisely because it is able to account for the cumulative effect of multiple variants (Ibanez et al., 2017). Indeed, it has been successfully used to identify at-risk individuals and measure their overall risk. The actual maximum prediction accuracy of PRS for Alzheimer's disease has been estimated to be of $74 \%$, compared to the $82 \%$ theoretical maximum that can be achieved by predictors of risk based on genotype data (Escott-Price et al., 2017). Mild cognitive impairment (MCI) is an intermediate stage in the progression from expected cognitive decline to dementia. Recently, an AD PRS has also been used to identify MCI in subjects in their 50s (Logue et al., 2018). Other AD PRSs, both including and excluding APOE, were able to predict the level and rate of memory decline (Marden et al., 
2016). The average PRS in Parkinson's patients is indirectly proportional to age at onset (Escott-Price et al., 2015). A PD PRS has also been associated with the motor and cognitive decline in patients (Paul et al., 2018), but not with the levels of $\alpha$-synuclein in CSF (Ibanez et al., 2017).

Ideally, a PRS could capture the combined effects of individual genetic variation in a single value, applicable in a clinical setting as an indicator of genetic susceptibility. Importantly, PRSs remain constant throughout life, which enables prediction from any age. Early identification of individuals at increased risk is imperative, because the pathological processes of $\mathrm{AD}$ and $\mathrm{PD}$ begin long before symptom onset. For example, abnormalities in biomarker measurements are seen as early as 20 years before AD onset (Masters et al., 2015). Additionally, PRSs could also be useful to assess several aspects of disease progression, as shown by some of the studies described above, which could help to define disease prognosis and treatment options. Furthermore, a better understanding of the relationship between genotype and clinical phenotypes could also inform patient selection for clinical trials and other research endeavors to accelerate the development of more effective targeted therapies (Tan \& Jankovic, 2006).

It must be noted that, even though PRSs are clearly attractive for clinical implementation, their accuracy is not yet significant enough. PRSs will potentially become more informative with larger GWAS and knowledge of more variables (Ibanez et al., 2017). Conversely, other studies in AD indicate the contributions of any new findings are likely to be small and thus unnecessary for the overall genetic prediction of disease (Escott-Price et al., 2017). Either way, the accuracy of PRS may be improved through the combination with clinical information and environmental risk factors (Lewis \& Vassos, 2017).

Neither PRSs or any other forms of genetic testing are currently part of the routine diagnostic process for $\mathrm{AD}$ and PD. However, this might very well change in a not so distant future as the field continues evolving. The correct implementation of these tools will largely depend on the available legal safeguards worldwide, which is why we shall discuss their implications next. For the purpose of the ethical discussion in this manuscript, "positive results" and "risk-positive" will refer to high predisposition for a disease according to a genetic test.

\section{Social impacts of genetic testing: insights from the ecological model}

The social ecological model is a theory initially used to understand behavior (Bronfenbrenner, 1977). This systems approach to human development sees behavior as affected by - and affecting - different levels of the social environment. The ecological model has been used in health promotion (McLeroy, Bibeau, Steckler, \& Glanz, 1988) and enrolment in clinical trials (Salihu, Wilson, King, Marty, \& Whiteman, 2015), 
amongst other applications. In these cases, the model considers the following levels of influence: intrapersonal, interpersonal, institutional, community, and that of public policy (McLeroy et al., 1988).

The ecological model approach could be taken to help identify determinants of health related to the development of neurodegenerative disorders (Shi \& Zhong, 2014). In this paper, we shall be applying it to analyze the social, ethical and legal implications of genetic testing for AD and PD in a broader social sense. At the intrapersonal or individual level, we find a participant's understanding of basic biological concepts and their reaction to results. The sphere of family at the interpersonal level can motivate an individual's decision to get tested if, for example, there is a known history of neurodegenerative disease in the family. Conversely, a positive result for $\mathrm{AD}$ or $\mathrm{PD}$ may have implications for the relatives of an individual who gets a genetic test. The institutional level includes centers carrying out research on genetic markers and insurance companies, while the community level emphasizes the influence of the cultural context on attitudes toward genetic testing or neurodegenerative diseases themselves. Finally, the public policy level is defined by the local legislation and international treaties.

Rather than focus on individuals as separate entities, the implications of any technological development must be discussed in the context of the interdependency amongst individuals, their relationships and their environments. Using the ecological model to understand the ramifications of genetic testing for late-onset diseases can better inform its implementation and regulation. Ultimately, the ecological model can also help devise strategies to influence public attitudes toward these new technologies.

\section{Ethical considerations associated with susceptibility genetic testing for neurodegenerative disease}

The debate about the issues of genetic testing covers everything from the research which enables the creation of the technology to what uses are made of its results. Some relevant questions include: Who should have access to genetic testing, and under which conditions? Could genetic testing be mandatory under any circumstance? Should genetic test results ever be released to third parties, including the user's family? How can individuals be protected from unfair treatment because of their genetic status? These and other questions can be approached from four basic ethical principles: autonomy, non-maleficence, beneficence, and justice (Beauchamp \& Childress, 2001). These four concepts are common in bioethical debate and must be respected for any technological development to be considered ethical.

\subsection{Autonomy}

This principle, also referred to as respect for persons, can be simply defined as "self-determination, selfrule, or self-governance" (Institute of Medicine Committee on Assessing Genetic Risks [CAGR], 1994). Individuals are autonomous to the extent that they are able to make willing and reasoned choices and take 
action without outside control. Autonomy has been legally, socially and morally recognized. In the context of genetic testing, respect for autonomy can be summed up in the right of individuals to make informed decisions on whether they wish to be tested, and what they wish to do with the results of said tests.

\subsubsection{Informed consent}

Informed consent ascertains that individuals make decisions in a free, voluntary and rational manner. In the case of genetic tests, this is only achieved when users understand the purpose, risks, benefits, alternatives and possible outcomes of the test, treatment options, and their own right to confidentiality and voluntary withdrawal (World Health Organization [WHO], 2006). In addition, there should be disclosure of any potential conflicts of interest, such as financial interest or patents (CAGR, 1994). These same basic conditions apply for participation in research and clinical trials prior to or following a genetic test, with the addition of specific requirements to each case.

When acquiring informed consent in a research setting, one major issue is clarifying that participation does not necessarily result in a direct benefit. Most experimental protocols will include control groups, which receive a known effective treatment or, if none exists, a placebo. Subjects wishing to participate in a trial will presumably do so with the hope of improving their condition or, in the case of prevention trials, avoid disease development. However, they must understand that they might be placed in a control group, where results are expected to be inferior than the experimental group. Knowing this information might dissuade some individuals from enrolling in a trial and, in the case of placebo protocols, might defeat the purpose if the effect is lost. Even so, individuals must be allowed to make their choice based on their own assessment of risks and benefits. In addition, group placement must be done randomly so that all enrolled subjects have the same fair chance of receiving treatment.

Meeting even just the minimum requirements for an informed consent form proves difficult with many recent technologies, such as screening for multiple disorders at once. Communicating all the possible outcomes and treatment options for each disease to the user might be more information than an individual can assimilate at once. Additionally, genetic test providers in a globalized world must ensure that informed consent forms are suitable for users from diverse cultural backgrounds. It is important to note that both providers and clinicians who refer patients to testing, have the duty to find alternatives themselves when faced with obstacles applying the traditional mechanisms of consent; the right to be informed must be respected first and foremost (CAGR, 1994). Collective experiences on what users of these new technologies want and need to know will be fundamental to legally safeguard their autonomy (Roche \& Berg, 2015). 
It is important to emphasize that there exists an intrinsic power imbalance in situations where genetic testing is involved. Participants in a clinical trial, for example, have less technical knowledge than the researchers, placing them in a position of vulnerability. This imbalance may be aggravated when taking into account the gender, ethnicity, socioeconomic status, or other social conditions of a subject. AD and PD patients, for example, are vulnerable given their dependency on others. Informed consent is a tool designed precisely to empower individuals in a position of vulnerability, and to ensure that their decision is not in any way coerced. However, this process is hindered when, in addition to the pre-existing imbalance, the subject of interest is an agent with decreased autonomy. How can the fully informed consent of these individuals be ensured, or should their use of genetic tests and participation in research be limited? This question is extremely controversial, since the complete exclusion of these groups from research and denial of health services could be considered a form of discrimination.

For example, some patients likely to be tested for susceptibility to a neurodegenerative disease may already be suffering from cognitive difficulties, compromising their full understanding of the test (Roberts \& Uhlmann, 2013). A legal proxy might be asked to be involved in the process to make a decision. However, it is hard to determine whether they will act in accordance with the user's wishes. The same issue arises in relation to genetic testing of underage individuals, where adults might act as legal proxies. According to the International Declaration on Human Genetic Data, genetic testing of children or adults unable to consent is only ethically acceptable if (i) the results have important implications for the health of the individual and (ii) the test takes into account their best interest (United Nations Educational, Scientific and Cultural Organization [UNESCO], 2003). AD and PD patients with a compromised capacity for free consent should be protected against abuse, and their personal integrity respected (UNESCO, 2005).

There are further ethical dilemmas specific to genetic testing of underage individuals. The best interest of the child should always outweigh any interest of their parents or legal tutors, as indicated in the Convention on the Rights of the Child (United Nations General Assembly, 1989). In the case of susceptibility testing for diseases such as $\mathrm{AD}$ and $\mathrm{PD}$, for which results are probabilistic and there are no cures or preventive measures available, it would be difficult to argue the existence of any benefits to a child knowing their genotype (Goldman et al., 2011). Furthermore, positive test results might trigger anxiety or depression in the child, or even cause a sense of guilt in the parents due to the hereditary component (Cozaru et al., 2016).

There might be an argument in favor of genetic testing if the parents' reasoning is for financial or life planning in future benefit of the child. Another argument in favor is that neurodegenerative diseases with late onset allow for many years of a healthy life before the child would present any of the debilitating 
symptoms. This is particularly relevant, considering the rapid rate at which knowledge is generated: it is likely that there will be more treatment options available by the time onset occurs.

Nevertheless, this is mere speculation and, as a general rule, there exists a consensus in that the principle of autonomy requires waiting until the child is able to make an independent decision (Paulsen et al., 2013). It is important to note that this should not necessarily mean to wait for the individual to legally become an adult. The principle of progressive autonomy is understood as the constantly evolving capacity of a child over time (Brems, Desmet, \& Wouter, 2009). According to this approach, both parents and healthcare providers should facilitate and support children's right to exercise their autonomy in relation to genetic testing. This can be achieved through informed assent, which is obtained adapting language according to the child's age, and employing other tools in addition to written forms, such as visual aids.

\subsubsection{Disclosure of results and unexpected findings}

Individuals have the right to decide what they want or do not want to know about their results. This right is protected by national and international legislation around the world (Pont-Sunyer et al., 2015). Decisions related to learning about several kinds of information are deeply personal and depend on a large variety of factors, including cultural and economic ones. However, sometimes it might be justified to override a previously stated preference for non-disclosure, depending on whether the condition is serious, treatable, or represents a risk to others. This last issue relates to whether the right to autonomy has more value than the right to health. We argue that, since health is defined as more than just physical well-being, an individual's informed analysis of what constitutes their own well-being should prevail.

In the case of a genetic test providing information about multiple disorders at once, the assessment of the risks and benefits of learning a result could differ between those relevant to the original purpose and secondary findings. Furthermore, the decision to know would probably depend on the nature of the secondary finding: a medically actionable condition or one without a cure; a progressive degenerative disease or a stable one (Roche \& Berg, 2015). Importantly, new forms of sequencing make it difficult to clearly define 'unexpected' or 'incidental' findings, notably in the context of research and clinical trials (Christenhusz, Devriendt, \& Dierickx, 2013). The process of informed consent is fundamental in order to ensure respect of the individual's autonomy regarding the disclosure of results, and updated mechanisms of consent are imperative for these new technologies. For example, the user should be able to make choices based on general categories, such as choosing to refrain from being informed about certain diseases before even being tested (CAGR, 1994). 
It is important to point out that non-disclosure can work differently in a research setting (i.e. clinical trials), in contrast to patients being referred to a genetic test by their clinician. For example, some protocols might explicitly require results not to be divulged to the participants in order to eliminate bias. Another issue related to the particular context of research arises in prevention trials, where the question is not whether researchers wish to disclose results, but rather if participants want to know.

In prevention trials, the subjects of interest are those presenting risk markers but who show no symptoms of disease. It stands to reason that people who wish to participate in a trial do not necessarily want information about their genetic status. In prevention trials, enrolment protocols are said to be blinded when they accommodate the choice of not knowing (Kim, Karlawish, \& Berkman, 2015). For example, they include risk-negative subjects in the study so that participation does not automatically equate to being a carrier. In contrast, transparent enrolment protocols do not consider the option of non-disclosure, and most trials of these type only involve risk-positive subjects (Kim et al., 2015). Some critics of transparent enrolment pose that such protocols are coercive, arguing that blinded enrolment is a requisite for autonomy. Nevertheless, others argue that blinded enrolment is not ethically required, and point out that transparent protocols could in fact be of greater benefit, since it spares risk-negative subjects from the burden of participation without any personal gain (Kim et al., 2015).

\subsubsection{Mandatory versus voluntary screening}

Genetic screening is defined as large-scale systematic testing offered within a program intended to detect genetic characteristics in asymptomatic people (UNESCO, 2003). There is no inherent moral argument in favor or against genetic screening and monitoring; instead, the value depends on how the tests are carried out and how the results are used (Congress of the United States Office of Technology Assessment, 1983). According to the Additional Protocol to the Convention on Human Rights and Biomedicine concerning Genetic Testing for Health Purposes (AP-GTHP, 2008), the following criteria must be met before any genetic screening program is implemented: (i) independent evaluation of its ethical acceptability; (ii) approval from the competent body; (ii) the program is recognized for its health relevance for the whole population or section of population concerned; (iii) the scientific validity and effectiveness of the program have been established; (iv) appropriate preventive or treatment measures in respect of the disease or disorder are available; (v) appropriate measures are provided to ensure equitable access to the program; (vi) the program provides measures to adequately inform the population or section of population concerned of the existence, purposes and means of accessing the screening program as well as the voluntary nature of participation in it. Additionally, the program should be supported by public education and genetic counselling (WHO, 2006). 
While it is recognized that the decision to participate in any genetic test must be voluntary, some decisionmakers might argue in favor of a mandatory testing in some cases. This due to the fact that it is a minimally invasive procedure whose results could contribute to a better resource allocation and a more efficient public health planning. For example, assessing an individual's PRS in relation to the overall population distribution allows the identification of the percentiles most and least at risk of developing a disease (Lewis \& Vassos, 2017). A PRS-based population screening might aid public health programs to strategically invest on those who need it. Individuals with a high PRS might be offered regular screening, referred to clinical trials, encouraged to change modifiable behaviors to decrease risk, or receive treatment when available (Lewis \& Vassos, 2017).

Arguing in favor of collective rights over the culture of individual rights inherent to autonomy, some commentators suggest measures such as mandatory genetic screening to warn people of their risk of genetic disorders (CAGR, 1994). In contrast to infectious diseases with immediate detrimental effects, for which the collective approach is usually taken, genetic disorders create a potential risk for futures generations (CAGR, 1994). However, in the case of late-onset neurodegenerative disorders, the risk is already clear in terms of increasing social and economic burden, following the rise of the prevalence of these conditions in coming years.

A manner of mandatory screening might be prenatal susceptibility testing. Testing for AD and PD is more technically complicated than for other conditions, such as some monogenic diseases. Therefore, prenatal testing is usually strongly discouraged (Tan \& Jankovic, 2006), especially if the user intends to carry a pregnancy to term regardless of the results (Goldman et al., 2011). In fact, requests for prenatal diagnosis of late-onset diseases are uncommon (Farlow et al., 2004), which indicates that a mandatory program would not be well received by the public. However, these views might change as technologies become more reliable. For example, preimplantation genetic diagnosis has already been used to ensure the implantation of familial AD risk-free embryos (Verlinsky et al., 2002).

The issue of prenatal screening is further complicated by the matter of abortion, even without considering mandatory abortion programs. Since there are no preventive measures available for AD and PD, the question arises if prenatal testing is a way to avoid the birth of particular individuals with the disease, rather than to prevent the conditions themselves (CAGR, 1994). Medical professionals have many different perspectives regarding genetic testing which could influence the termination of a pregnancy (Farlow et al., 2004). Regardless of personal beliefs, in countries where the legal right to elective abortion has been granted, selective abortion is an available option. 
It is important to note that legal rights do not resolve the related ethical dilemmas. Selective abortion in these cases is based on maternal considerations of the future child's quality of life (Post, 1994). While it is exclusively a woman's choice to terminate a pregnancy, several factors can contribute to the decision. There is a great variation in what people regard as a worthy and healthy lifestyle, which can be influenced by possible stigma towards disease and limited understanding of genetics. In conditions with variable expressivity, uncertain manifestation, and late onset times, some argue that it becomes difficult to establish a solid moral argument in favor of selective abortion (Post, 1994).

The use of assisted reproduction technologies with the purpose of choosing a future child's sex is prohibited, except when a serious sex-related disease can be avoided (Convention on Human Rights and Biomedicine [CHRB], 1997). This safeguard aims to discourage gender-based discrimination. In that sense, mandatory prenatal screening for a non-deterministic predisposition for PD or AD could be viewed as a form of discrimination. If the only aim of a mandatory screening program were as a warning, legislating against selective abortion based on the results could be a necessary protection against discrimination. However, that would limit women's right to choose. The answer would be to provide thorough information about the implications of a risk-positive result, particularly for couples with a clinical history of genetic disease (Brazier \& Cave, 2011). This way, the effect of outside factors on the woman's decision are minimized.

Even with the safeguard of information, it is still difficult to justify a mandatory screening program, regardless of whether it is planned for adults or as prenatal testing. It is undeniable that the onus of caring for patients with $\mathrm{AD}$ and $\mathrm{PD}$ will greatly affect future generations. It is also important to note that some societies value collective over individual benefits because of their cultural context, and this should be taken into account when weighing the ethical merit of such measures. However, we argue that the promise of social long-term benefits is not sufficient justification for disregarding the individual right to choose, especially given the nature of genetic information.

\subsubsection{Privacy and confidentiality}

Following the idea that an autonomous individual holds power over their own self, then they must have the right to use restrictive measures for their protection. Privacy and confidentiality extend to the right not to disclose personal information, such as the results of genetic testing, to third parties (WHO, 2006). According to internationally recognized declarations, a person's biological information must be held confidential in the conditions set by law and should not be disclosed or made accessible to third parties (i.e. employers, insurance companies, educational institutions, or the family) (UNESCO, 2005). This umbrella of protection covers human genetic data, human proteomic data, and biological samples associated with an identifiable person, stored or processed for the purposes of medical genetic tests, research or any other 
purpose (UNESCO, 1997; UNESCO, 2003). The only exceptions for disclosure considered by international instruments are those of public interest, with restrictions stated by domestic law consistent with the international law of human rights. The other exception relates to cases when the person concerned has provided prior, free, informed and express consent for disclosure. Additionally, individuals are granted the right to respect for their private life, in particular to protection of their personal data derived from a genetic test (AP-GTPH, 2008).

Challenging these ideas, some argue that genetic testing not only reveals the risk to an individual, but also to blood relatives, raising the question of how personal genetic information actually is. Additionally, a positive result can have considerable consequences for spouses (i.e. reproductive decisions) and other members of the patient's close social context (e.g. potential caregivers). As such, disclosing genetic risk might be considered a moral obligation. However, ethical reasoning should be made in the context of the genetic complexity of a specific condition (Arribas-Ayllon, 2011). In the case of AD and PD, for which genetic tests are largely probabilistic, there is no clear benefit of disclosure to family members.

\subsection{Non-maleficence and beneficence}

Respecting choice is not enough for the ethical treatment of a person Efforts should also be made to secure their well-being a protect them from harm (National Commission for the Protection of Human Subjects of Biomedical and Behavioral Research [NC-PHS], 1979). In this sense, non-maleficence and beneficence are closely related concepts dealing with the principles of maximizing benefits while minimizing risks (UNESCO, 2005). These principles are relevant primarily to the interpretation of the results of a genetic test, and the disclosure of this information to third-parties.

\subsubsection{Stigma and genetic discrimination}

Indiscriminate access to medical and genetic information these data could give rise to different forms of discrimination and risks to the individual. For this reason, safeguards for privacy and confidentiality are extremely important. Knowledge of a person's genetic status can cause stigma depending on their context. For example, in low- and middle-income countries, dementia is often regarded as a normal part of ageing (ADI, 2010). However, a known positive result for AD or PD in other contexts could also lead to the individual being belittled, even before the appearance of symptoms.

Beyond stigmatization, the disclosure of a person's genetic information can lead to discriminatory behavior. Genetic discrimination involves the differential treatment of a person on the grounds of their actual or presumed genetic differences, known either through genetic testing, family history, or even information about the communities they belong to (Otlowski, 2005). However, a person's identity should not be reduced 
to genetic characteristics, since it involves complex educational, environmental and personal factors, along with emotional, social, spiritual and cultural bonds (UNESCO, 2003). Several international legal instruments prohibit any form of discrimination based on genetic heritage in a way that infringes a person's basic human rights and fundamental freedoms, or for purposes that lead to the stigmatization of an individual, a family, a group, or a whole community (CHRB, 1997; UNESCO, 1997; UNESCO, 2003; UNESCO, 2005; AP-GTHP, 2008).

Genetic discrimination causes disadvantages with respect to access to health services, education and employment. In the United States, the Genetic Information Nondiscrimination Act (GINA, 2008) prohibits health insurers from denying coverage or charging higher premiums based solely on a genetic predisposition. It also prohibits employers from using a person's genetic information as deciding factors for decisions such as hiring, firing, assigning jobs, or any other terms of employment. Finally, it prohibits employers from requesting, requiring, or purchasing genetic information about their employees and their family members (Hudson, 2011). A number of developing countries have also introduced legislative measures to protect citizens against discrimination and stigmatization related to medical conditions and genetic status (WHO, 2006).

Regarding late-onset diseases, only a few people in some surveys express concerns over third-party access to their genetic status, which could indicate that information about conditions such as AD and PD is perceived as less likely to affect employment or insurance (Neumann, 2001). Even if that were the case, measures must still be taken to protect genetic information related to these diseases because of the overall sensitive nature.

\subsubsection{The importance of genetic counselling}

Another concern of genetic testing is the impact on the individual's self-image. Unlike infectious disease, genetic disease can be considered a part of the patient's intrinsic identity, leading to self-classification as "defective" (CAGR, 1994). A test confirming, or even suggesting, the future development of a neurodegenerative condition can be overwhelming for a patient. Genetic test results for AD and PD, particularly if unexpected, may trigger adverse psychological responses, including stress and severe depression (Goldman et al., 2011; The Michael J. Fox Foundation for Parkinson's Research, 2018). Conversely, not having clear answers is also a source of anxiety (Cozaru et al., 2016). Disclosure of genotyping information can actually represent a benefit for those negative, and even for risk-positive individuals, as long as some constraints are established (Green et al., 2009). The main and most relevant requirement is the provision of appropriate counselling by trained professionals. 
Genetic counselling is essential to preserve autonomy in the informed consent process prior to testing, and it represents the difference between favorable and adverse reactions to the results following the test. In the case of complex diseases like $\mathrm{AD}$ and $\mathrm{PD}$, genetic counselling should be done on a case-by-case basis, in order to account for the context of the individual (Farlow et al., 2004). When proper post-test counselling is guaranteed, there exists no further psychological risk. Some studies argue that genotype disclosure does not even result in short-term psychological problems (Ramani \& Saviane, 2010). Research demonstrates that using standardized counselling protocols after an AD susceptibility test ensures effective coping skills (Goldman et al., 2011). Similarly, asymptomatic testing for PD, when done with the appropriate genetic counselling, may be useful to ease the anxiety of an at-risk individual when negative, or help them cope with transient distress when positive (Tan \& Jankovic, 2006).

The results of genetic testing can also influence a person's finances, reproductive planning, and interpersonal relationships (Cozaru et al., 2016). Genetic counselling is also important to avoid unreasoned decisions stemming from misunderstood technical terms, including the meaning of susceptibility itself. Some studies suggest that genetic risk information enhances preferences for potentially unnecessary medical interventions (Roberts, Christensen, \& Green, 2011). Conversely, they might encourage behavioral changes, increased awareness of the person's health, and even the purchase of long term insurance. This is particularly relevant for neurodegenerative disorders, because patients are often placed in nursing homes and go through lengthy inpatient stays (Roberts et al., 2011; Paulsen et al., 2013).

\subsection{Justice}

The concept of justice encompasses both the access and contribution to genetic technologies: all groups should share the benefits and burdens equally. There are several widely accepted formulations of just ways to distribute burdens and benefits: (i) to each person an equal share, (ii) to each person according to individual need, (iii) to each person according to individual effort, (iv) to each person according to societal contribution, and (v) to each person according to merit (NC-PHS, 1979). Distributive and contributive justice are relevant to public planning, including questions of fair allocation of limited resources, how to deal with competing needs, and how to ensure the accessibility to technologies.

\subsubsection{Diversity in clinical and biomedical research}

Even though non-discrimination is a key principle in human rights, health disparities are deeply connected to sectors of society which have been historically discriminated against (United Nations Office of the High Commissioner for Human Rights [OHCHR], 2008). In the past, research has failed to fairly represent minority populations in clinical trials, and in biomedical research specific to factors relevant to their health. 
The lack of diversity severely limits the overall understanding of pathology of diseases in which risk differs amongst ethnic groups, such as AD and PD (Goldman et al., 2011; Roberts \& Uhlmann, 2013; Marden et al., 2016; Poewe et al., 2017). Particularly, it hinders knowledge of the interactions between genetic status and certain environmental risks to confer a prognosis (Oh et al., 2015). Racial diversity is also necessary in GWAS to improve the predictive capability of PRSs, since markers for discovered in a population do not necessarily show the same association to disease in other groups (Marden et al., 2016).

A greater issue is that discrimination operates in a loop: Minority groups experience health disparities because of their racial and ethnic background. For this reason, they are excluded from clinical and biomedical research. Knowledge is developed based on information obtained from the largely homogenous population which does participate in research. Finally, implementation of technologies created from this knowledge has to be either extrapolated to the minority groups or they are restricted access completely, perpetuating the health disparities.

Particularly in a globalized world with rapidly changing demographics, representation of diversity in research is a necessary requirement for justice (Oh et al., 2015). All members of society should equally share in the burden of research (i.e. participating in a clinical trial) and equally reap the benefits (i.e. having access to genetic testing).

\subsubsection{Availability and accessibility of genetic tests}

Two essential requirements for a just distribution of healthcare are its availability and accessibility. The former refers to the sufficient quantity of goods and services, while the latter implies financial and nondiscriminatory access to healthcare. Accessibility also refers to the right to seek, receive and impart healthrelated information, without impairing the right to confidentiality of others (OHCHR, 2008).

Individuals have a right to enjoy the highest standards of health, in a system which provides equal opportunity for everyone (OHCHR, 2008). Whether such a system should consider access to genetic testing is debatable. In the case of $\mathrm{AD}$ and $\mathrm{PD}$, taking a susceptibility test does not determine the outcome of a positive or negative prognosis. However, early diagnosis of at-risk individuals allows the stratification of patients and, possibly, optimal placing in clinical trials according to their genetic status. The question is whether the chance of a more positive outcome, helped by a genetic test result, is sufficient to demand the accessibility of genetic testing, especially considering the implication of financial attainability. If there eventually exists a reliable therapeutic method for either AD ot PD, would that signify a stronger argument? Additionally, conditions with a hereditary component will have increased incidence in particular groups, in contrast to infectious disease which generally affect the entire population. If the accessibility of genetic 
testing for $\mathrm{AD}$ and $\mathrm{PD}$ is ensured, some might argue that this benefits only certain populations with the predisposition, aggravating inequity amongst these groups and "healthy" individuals. However, we hold that such inequities are morally permissible, since they are in the benefit of the least well-off group. In general, access to genetic testing could potentially improve benefits of public health policies and also expand the delineation of the principle of beneficence.

Some might argue that clinical and research settings obstruct, rather than facilitate, both the availability and accessibility of genetic testing. The marketing of direct-to-consumer (DTC) tests has emerged as an answer to the general public's desire to know as much about their genes and their bodies as they choose, through simple and affordable services (Ramani \& Saviane, 2010). DTC tests aid availability by increasing the supply of genetic tests in the market, which in turn helps lower prices to make these services more accessible. However, another key aspect to consider for a fair allocation of healthcare is the quality: What is the scientific and medical suitability of the tests, and whose responsibility it is to ensure it? Currently, there are very few controls in place at global levels to assess the validity of tests before they are marketed (Kaye, 2008). In Europe, some legislation requires the clinical utility of genetic tests to be determined previous to marketing (AP-GTHP, 2008). Similarly, the Food and Drug Administration in the United States called for the need to prove validity of already marketed tests, on the grounds that they are diagnostic tools; however, companies were not required to stop selling their products (Ramani \& Saviane, 2010).

While the importance of consumer choice must be recognized, and a re-evaluation of the way genetic testing is currently carried out will be beneficial in the long run, consumer protection cannot be disregarded (Kaye, 2008). DTC testing for multiple diseases at once gives consumers a relative risk for each condition as compared to the general population; however, results do not report an absolute risk, because companies lack information relative to family history and environmental risks (Kaye, 2008). DTC testing companies do not offer direct genetic counselling by healthcare professionals which, as previously discussed, could lead to psychological distress if the individual does not understand the significance, or lack thereof, of the results provided (Roberts et al., 2011).

Regarding the accessibility of information, some researchers pose that places with higher levels of education and scientific literacy, more awareness about science and technology, and advanced political debate, are the countries in which people are more skeptical about genetic testing (Ramani \& Saviane, 2010). For example, Nordic and Dutch populations, which are 'attentive' to developments in biotechnology - that is, have high levels of understanding of basic biology and genetic concepts, have read or heard about biotechnology in the mass media, and talk about these topics with others -, are less likely to take a genetic test to detect a disease (European Commission [EC], 2006). Similarly, Austria and Italy, where willingness 
to get tested for a disease is as low as $45 \%$ and $62 \%$ of the public respectively, have populations considered 'active' in the public debate regarding biotechnology (EC, 2006). This might suggest that the more people understand the issues, the less enthusiastic they are about new technologies. However, countries with populations considered to be 'unengaged' in issues concerning biotechnology and genetics, can be either very highly interested (e.g. Portugal, 81\%) or very uninterested (e.g. Poland, 59\%) in genetic testing for disease (EC, 2006). This shows that, even though there is some correlation between knowledge and participation, the public attitude towards genetic testing is also defined by other factors, such as the cultural context.

Even so, it is imperative that the potential users of genetic testing are properly guided by the medical community (Tan \& Jankovic, 2006). Before being able to properly educate the general public, professional healthcare providers must first and foremost understand: (i) how easily misinterpreted probabilistic estimates of risk can be in the absence of family history or information about environmental risks, and (ii) that said misinterpretations can have varying harmful effects depending on several contextual factors of the patient. The fact remains that the understanding of risk amongst the medical community is exceedingly low (Green et al., 2009). For PRSs, the concept of genetic susceptibility based on a continuous score, instead of on the presence/absence of a high-risk variant, also entails a paradigm shift for medical professionals in their understanding of genetic disorders (Lewis \& Vassos, 2017). Sharing knowledge with laypersons is further hindered by the lack of education in areas like nonverbal and intercultural communication. The remedy is to teach doctors how to communicate more effectively (Brazier \& Cave, 2011). Baseline knowledge of basic genetics principles, and interest in and opinions regarding genetic testing should be assessed to develop appropriate educational materials for both healthcare providers and the general public (Falcone, Wood, Xie, Siderowf, \& Van Deerlin, 2011). It is imperative to mention that the onus in both cases falls mainly to public institutions and public health models to promote a more integral education of the population.

\subsubsection{Allocation of resources}

Genetic testing raises questions of justice in terms of allocation of resources. The experience with DTC tests suggests that the demand for testing is considerable and might be even greater if costs decreased further. Currently, the price of genetic testing is anywhere from less than 100 to more than 2000 US dollars (National Human Genome Research Institute, 2017). Considering that the prevalence of neurodegenerative diseases will continue rising, information on genetics of $\mathrm{AD}$ and $\mathrm{PD}$ is likely to affect a much wider population (Post, 1994). Since those with high levels of perceived risk (i.e. because of family history or caring for a patient) are most likely to pursue genetic testing (Wikler, Blendon, \& Benson, 2013), demand 
will presumably increase, given that more people will have close proximity to patients within their social environment.

If a significant number of consumers use their genetic testing to inform investment of long-term care insurance, it might be argued that, as businesses, insurance companies could increase premiums based on susceptibility to AD or PD. We discussed above that this would be considered genetic discrimination. However, a lot of legislation in the matter only covers health insurance and not long-term care insurance or other domains (Roberts et al., 2011).

As previously stated, another question arises: Will the increasing supply and demand of genetic tests for a growing number of conditions push governments to provide the service as part of a public health program? Even if prices for genetic testing decrease, cumulative costs would still represent a significant sum, especially if genetic counselling is also provided. Additionally, resources are limited, and decision-makers need to take as many factors as possible into consideration when budgeting. For example, would widespread investment on genetic disease raise health costs? If not, it would be due to a decrease in budget for another area (e.g. infectious disease), because every expense has an associated opportunity cost. Even if public expenditure on genetic testing is approved, late-onset neurodegenerative diseases would most likely be of low priority, at least until treatment is available or the burden becomes more obvious.

\section{Conclusions}

Beyond understanding the biology of disease, some of the ultimate goals of research in genomics include advancing the science of medicine and improving the effectiveness of healthcare. Undoubtedly, integration poses great challenges. However, it is an ethical obligation to make the best use possible of scientific progress to advance society (Brand, Brand, \& Schulte in den Bäumen, 2008).

In the case of genetic testing for late-onset neurodegenerative disorders, a plethora of issues arises. What we have discussed in this manuscript is just tip of the iceberg: we have not touched upon matters related to, for example, advanced requests for euthanasia and physician-assisted suicide after early diagnosis of neurodegenerative disease. However, we believe that we have established the importance of governments strengthening legislation to protect against genetic discrimination and to support the principle of autonomy. Public health actions should address these questions in order to reduce the negative consequences of neurodegenerative disorders. In addition to analyzing the issues of genetic technologies, increasing the investment on community care facilities and launching campaigns to educate the public are other valuable strategies. 
Even though evidence should be the base for science policy, trust will only be strengthened if public participation is taken into account in the discussion. It is imperative to bridge the gaps of communication amongst researchers, clinicians, decision-makers, and the general public. We must exploit the benefits of a public debate enriched by the multiple moral and philosophical points of view present in the pluralistic society of today's globalized world. Genetics research and technology will continue advancing and the only hope of catching up lies in the constant interdisciplinary discussion of the ethical, legal and social implications of science.

Acknowledgements: MER thanks the support of the Australian National Health \& Medical Research Council and the Australian Research Council through a NHMRC-ARC Dementia Research Development Fellowship (APP1102821).

Conflict of Interest: The authors declare no conflict of interest.

\section{References}

Additional Protocol to the Convention on Human Rights and Biomedicine concerning Genetic Testing for Health Purposes, Strasbourg, 27 November 2008, Council of Europe Treaty Series, No. 203. Retrieved from https://www.coe.int/en/web/conventions/full-list/-/conventions/treaty/203

Alzheimer's Disease International. (2010). World Alzheimer Report 2010: The Global Economic Impact of Dementia. Retrieved from https://www.alz.org/documents/national/world_alzheimer_report_2010.pdf

Arribas-Ayllon, M. (2011). The ethics of disclosing genetic diagnosis for Alzheimers disease: Do we need a new paradigm?. British medical bulletin, 100, 7-21. doi:10.1093/bmb/ldr023

Beauchamp, T. L., \& Childress, J. F. (2001). Principles of biomedical ethics. Retrieved from: https://books.google.com.mx/books?id=_14H7MOw104C\&dq=beauchamp+and+childress+principles+o $\underline{\mathrm{f}+\text { biomedical+ethics } \& \mathrm{lr}=\& \text { source }=\text { gbs navlinks } \mathrm{s}}$

Brand, A., Brand, H., \& Schulte in den Bäumen, T. (2008). The impact of genetics and genomics on public health. European Journal of Human Genetics, 16, 5-13. doi:10.1038/sj.ejhg.5201942

Brazier, M., \& Cave, E. (2011). Medicine, Patients and the Law (5th ed.). Penguin Books. 
Brems, E., Desmet, E., \& Wouter, V. (2009). Children's Rights Law in the Global Human Rights Landscape: Isolation, inspiration, integration? Retrieved from https://books.google.com.mx/books?id=C0AlDwAAQBAJ\&dq=progressive+autonomy+child\&source= gbs navlinks $\mathrm{s}$

Brofenbrenner, U. (1977). Toward an Experimental Ecology of Human Development. American Psychologist, 32, 513-531. doi:10.1037/0003-066X.32.7.513

Christenhusz, G. M., Devriendt, K., \& Dierickx, K. (2013). To tell or not to tell? A systematic review of ethical reflections on incidental findings arising in genetics contexts. European Journal of Human Genetics, 21, 248-255. doi:10.1038/ejhg.2012.130

Congress of the United States Office of Technology Assessment. (1983). Chapter 9: Application of Ethical Principles to Genetic Testing. In The Role of Genetic Testing in the Prevention of Occupational Disease (pp. 139-148). Retrieved from https://www.princeton.edu/ ota/disk3/1983/8317/831711.PDF

Convention for the Protection of Human Rights and Dignity of the Human Being with regard to the Application of Biology and Medicine: Convention on Human Rights and Biomedicine, Oviedo, 4 April 1997, European Treaty Series, No. 164. Retrieved from https://www.coe.int/en/web/conventions/full$\underline{\text { list/-/conventions/treaty/164 }}$

Cozaru, G. C., Aşchie, M., Mitroi, A. F., Poinăreanu, I., \& Gorduza, E. V. (2016). Ethical and genetic aspects regarding presymptomatic testing for neurodegenerative diseases. Revista medico-chirurgicala $a$ Societatii de Medici si Naturalisti din Iasi, 120, 15-22.

de Lau, L. M., \& Breteler, M. M. (2006). Epidemiology of Parkinson's disease. Lancet Neurology, 5, 525535. doi:10.1016/S1474- 4422(06)70471-9

Duthey, B. (2013). Background paper 6.11: Alzheimer disease and other dementias. In Priority Medicines for Europe and the World "A Public Health Approach to Innovation" Update on the 2004 Background Paper (pp. 6.11-1-6.11-71) World Health Organization. Retrieved from http://www.who.int/medicines/areas/priority medicines/BP6 11Alzheimer.pdf

Escott-Price, V., Nalls, M. A., Morris, H. R., Lubbe, S., Brice, A., Gasser, T. ... Williams, N. M. (2015). Polygenic risk of Parkinson disease is correlated with disease age at onset. Annals of Neurology, 77, 582591. doi:10.1002/ana.24335 
Escott-Price, V., Shoai, M., Pither, R., Williams, J., \& Hardy, J. (2017). Polygenic score prediction captures nearly all common genetic risk for Alzheimer's disease. Neurobiology of Aging, 49, 214.e7214.e11. doi:10.1016/j.neurobiolaging.2016.07.018

European Commission. (2006). Europeans and Biotechnology in 2005: Patterns and Trends. Retrieved from http://ec.europa.eu/public_opinion/archives/ebs/ebs_244b_en.pdf

Falcone, D. C., Wood, E. M., Xie, S. X., Siderowf, A., \& Van Deerlin, V. M. (2011). Genetic Testing and Parkinson Disease: Assessment of Patient Knowledge, Attitudes, and Interest. Journal of Genetic Counseling, 20, 384-395. doi:10.1007/s10897-011-9362-0

Farlow, J., Pankratz, N. D., Wojcieszek, J., \& Fouroud, T. (2004, May 25). Parkinson Disease Overview. In M. P. Adam, H. H. Ardinger, R. A. Pagon, \& S. E. Wallace (Eds.), GeneReviews ${ }^{\circledR}$. Retrieved from https://www.ncbi.nlm.nih.gov/books/NBK1223/

Findley, L. J. (2007). The economic impact of Parkinson's disease. Parkinsonism \& Related Disorders, 13(Suppl), S8-S12. doi:10.1016/j.parkreldis.2007.06.003

Genetic Information Nondiscrimination Act of 2008, Pub.L. 110-233, 122 Stat. 881 (2008). Retrieved from https://www.gpo.gov/fdsys/pkg/PLAW-110publ233/content-detail.html

Goldman, J. S., Hahn, S. E., Catania, J. W., LaRusse-Eckert, S., Butson, M. B., Rumbaugh, M., ... Bird, T. (2011). Genetic counseling and testing for Alzheimer disease: Joint practice guidelines of the American College of Medical Genetics and the National Society of Genetic Counselors. Genetics in Medicine, 13, 597-605. doi:10.1097/GIM.0b013e31821d69b8

Green, R. C., Roberts, J. S., Cupples, L. A., Relkin, N. R., Whitehouse, P. J., Brown, T., ... Wright, L. (2009). Disclosure of APOE Genotype for Risk of Alzheimer's Disease. The New England Journal of Medicine, 361, 245-254. doi:10.1056/NEJMoa0809578

Hudson, K. L. (2011). Genomics, Health Care, and Society. The New England Journal of Medicine, 365, 1033-1041. doi:10.1056/NEJMra1010517

Ibanez, L., Dube, U., Saef, B., Budde, J., Black, K., Medvedeva, A., ... Cruchaga, C. (2017). Parkinson disease polygenic risk score is associated with Parkinson disease status and age at onset but not with alphasynuclein cerebrospinal fluid levels. BMC Neurology, 17, 198-206. doi:10.1186/s12883-017-0978-z

Institute of Medicine Committee on Assessing Genetic Risks. (1994). Social, Legal, and Ethical Implications of Genetic Testing. In L. B. Andrews, J. E. Fullarton, N. A. Holtzman, \& A. G. Motulsky 
(Eds.), Assessing Genetic Risks: Implications for Health and Social Policy (pp. 247-289). Retrieved from https://www.nap.edu/read/2057/chapter/10

Kalia, L. V., \& Lang, A. E. (2015). Parkinson's disease. Lancet, 386, 896-912. doi:10.1016/S01406736(14)61393-3

Kaye, J. (2008). The Regulation of Direct-to-Consumer Genetic Tests. Human Molecular Genetics, 17, R180-R183. doi:10.1093/hmg/ddn253

Kim, S. Y. H., Karlawish, J., \& Berkman, B. E. (2015). Ethics of genetic and biomarker test disclosures in neurodegenerative disease prevention trials. Neurology, 84, 1488-1494. doi:10.1212/WNL.0000000000001451

Lewis, C. M., \& Vassos, E. (2017). Prospects for using risk scores in polygenic medicine. Genome Medicine, 9, 96. doi:10.1186/s13073-017-0489-y

Logue, M. W., Panizzon, M. S., Elman, J. A., Gillespie, N. A., Hatton, S. N., Gustavson, D. E., ... Kremen, W. S. (2018). Use of an Alzheimer's disease polygenic risk score to identify mild cognitive impairment in adults in their 50s. Molecular Psychiatry. Advance online publication. doi:10.1038/s41380-018-0030-8

Marden, J. R., Mayeda, E. R., Walter, S., Vivot, A., Tchetgen Tchetgen, E. J., Kawachi, I., \& Glymour, M. M. (2016). Using an Alzheimer's Disease polygenic risk score to predict memory decline in black and white Americans over 14 years of follow-up Running head: AD polygenic risk score predicting memory decline. Alzheimer Disease and Associated Disorders, 30, 195-202. doi:10.1097/WAD.0000000000000137

Masters, C. L., Bateman, R., Blennow, K., Rowe, C. C., Sperling, R. A., \& Cummings, J. L. (2015). Alzheimer's disease. Nature Reviews Disease Primers, 1, 15056. doi:10.1038/nrdp.2015.56

McLeroy, K. R., Bibeau, D., Steckler, A., \& Glanz K. (1988). An ecological perspective on health promotion programs. Health Education Quarterly, 15, 351-377. doi:10.1177/109019818801500401

National Commission for the Protection of Human Subjects of Biomedical and Behavioral Research. (1979). The Belmont report: Ethical principles and guidelines for the protection of human subjects of research. Retrieved from: https://www.hhs.gov/ohrp/regulations-and-policy/belmont-report/read-thebelmont-report/index.html

National Human Genome Research Institute. (2017, July 5). Frequently Asked Questions About Genetic Testing. Retrieved from https://www.genome.gov/19516567/faq-about-genetic-testing/ 
Neumann, P. J., Hammitt, J. K., Mueller, C., Fillit, H. M., Hill, J., Tetteh, N. A., \& Kosik, K. S. (2001).

Public attitudes about genetic testing for Alzheimer's disease. Health Affairs, 20, 252-264. doi:10.1377/hlthaff.20.5.252

Oh, S. S., Galanter, J., Thakur, N., Pino-Yanes, M., Barcelo, N. E., White, M. J., .. Burchard, E. G. (2015). Diversity in Clinical and Biomedical Research: A Promise Yet to Be Fulfilled. PLoS Medicine, 12, e1001918. doi:10.1371/journal.pmed.1001918

Otlowski, M. J. (2005). Exploring the Concept of Genetic Discrimination. Journal of Bioethical Inquiry, 2, 165-176. doi:10.1007/BF02448597

Paul, K. C., Schulz, J., Bronstein, J. M., Lill, C. M., \& Ritz, B. R. (2018). Association of Polygenic Risk Score With Cognitive Decline and Motor Progression in Parkinson Disease. JAMA Neurology, 75, 360366. doi:10.1001/jamaneurol.2017.4206

Paulsen, J. S., Nance, M., Kim, J.-I., Carlozzi, N. E., Panegyres, P. K., Erwin, C., ... Williams, J. K. (2013). A Review of Quality of Life after Predictive Testing for and Earlier Identification of Neurodegenerative Diseases. Progress in Neurobiology, 110, 2-28, doi:10.1016/j.pneurobio.2013.08.003

Poewe, W., Seppi, K., Tanner, C. M., Halliday, G. M., Brundin, P., Volkmann, J., ... Lang, A. E. (2017). Parkinson disease. Nature Reviews Disease Primers, 3, 17013. doi:10.1038/nrdp.2017.13

Pont-Sunyer, C., Bressman, S., Raymond, D., Glickman, A., Tolosa, E., \& Saunders-Pullman, R. (2015). Disclosure of Research Results in Genetic Studies of Parkinson Disease Due to LRRK2 Mutations. Movement Disorders: Official Journal of the Movement Disorder Society, 30, 904-908. doi: $10.1002 / \mathrm{mds} .26250$

Post, S. G. (1994). Genetics, Ethics, and Alzheimer Disease. Journal of the American Geriatrics Society, 42, 782-786. doi:10.1111/j.1532-5415.1994.tb06542.x

Ramani, D., \& Saviane, C. (2010). Genetic tests: Between risks and opportunities. The case of neurodegenerative diseases. EMBO Reports, 11, 910-913. doi:10.1038/embor.2010.177

Rizzo, G., Copetti, M., Arcuti, S., Martino, D., Fontana, A., \& Logroscino, G. (2016). Accuracy of clinical diagnosis of Parkinson disease: A systematic review and meta-analysis. Neurology, 86, 566-576. doi:1212/WNL.0000000000002350

Roberts, J. S., \& Uhlmann, W. R. (2013). Genetic susceptibility testing for neurodegenerative diseases: Ethical and practice issues. Progress in Neurobiology, 110, 89-101. doi:10.1016/j.pneurobio.2013.02.005 
Roberts, J. S., Christensen, K. D., \& Green, R. C. (2011). Using Alzheimer's disease as a model for genetic risk disclosure: Implications for personal genomics. Clinical Genetics, 80, 407-414. doi:10.1111/j.13990004.2011.01739.x

Roche, M. I., \& Berg, J. S. (2015). Incidental Findings with Genomic Testing: Implications for Genetic Counseling Practice. Current Genetic Medicine Reports, 3, 166-176. doi:10.1007/s40142-015-0075-9

Salihu, H. M., Wilson, R. E., King, L. M., Marty, P. J., \& Whiteman, V. E. (2015). Socio-ecological Model as a Framework for Overcoming Barriers and Challenges in Randomized Control Trials in Minority and Underserved Communities. International Journal of MCH and AIDS, 3, 85-95.

Shi, Y., \& Zhong, S. (2014). From Genomes to Societies: A Holistic View of Determinants of Human Health. Current Opinion in Biotechnology, 28, 134-142. doi:10.1016/j.copbio.2014.03.001

Tan, E. K., \& Jankovic, J. (2006). Genetic Testing in Parkinson Disease: Promises and Pitfalls. Archives of Neurology, 63, 1232-1237. doi:10.1001/archneur.63.9.1232

The Michael J. Fox Foundation for Parkinson's Research. (2018, April). Navigating Clinical Trials: A Guide for Parkinson's Patients and Families. Retrieved from https://www.michaeljfox.org/files/PDEC_PATIENT_GUIDE.pdf

United Nations Educational, Scientific and Cultural Organisation. (1997). Universal Declaration on the Human Genome and Human Rights. Paris. Retrieved from http://portal.unesco.org/en/ev.php-

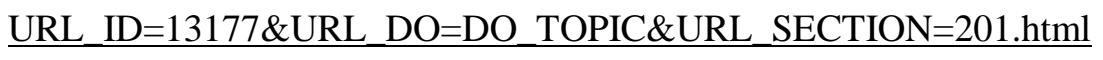

United Nations Educational, Scientific and Cultural Organisation. (2003). International Declaration on Human Genetic Data. Paris. Retrieved from http://portal.unesco.org/en/ev.php-

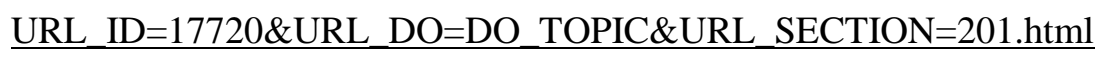

United Nations Educational, Scientific and Cultural Organisation. (2005). Universal Declaration on Bioethics and Human Rights. Paris. Retrieved from http://portal.unesco.org/en/ev.php-

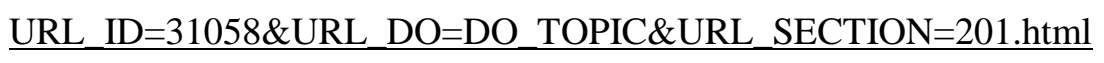

United Nations General Assembly. (1989). Convention on the Rights of the Child. New York City. Retrieved from http://www.ohchr.org/Documents/ProfessionalInterest/crc.pdf

United Nations Office of the High Commissioner for Human Rights. (2008, June). Fact Sheet No. 31: The Right to Health. Retrieved from http://www.ohchr.org/Documents/Publications/Factsheet31.pdf 
Verlinsky, Y., Rechitsky, S., Verlinsky, O., Masciangelo, C., Lederer, K., \& Kuliev, A. (2002) Preimplantation Diagnosis for Early-Onset Alzheimer Disease Caused by V717L Mutation. JAMA, 287, 1018-1021. doi:10.1001/jama.287.8.1018

von Campenhausen, S., Winter, Y., Rodrigues e Silva, A., Sampaio, C., Ruzicka, E., Barone, P., ... Reese, J. P. (2011). Costs of illness and care in Parkinson's disease: an evaluation in six countries. European Neuropsychopharmacology, 21, 180-191. doi:10.1016/j.euroneuro.2010.08.002

Wikler, E. M., Blendon, R. J., \& Benson, J. M. (2013). Would you want to know? Public attitudes on early diagnostic testing for Alzheimer's disease. Alzheimer's Research \& Therapy, 5, 43-53. doi:10.1186/alzrt206

World Health Organization. (2006). Medical genetic services in developing countries: the ethical, legal and social implications of genetic testing and screening. Geneva. Retrieved from http://apps.who.int/iris/handle/10665/43288 